\title{
The shifting role of animation in science dissemination
}

\author{
Keywords \\ Functional Animation, Interdisciplinary Collaboration, Science Dissemination, \\ Visualisation, Visual Science Communication.
}

This research proposal aims to map animation possibilities in science communication focusing on public science dissemination. The research plan is helped by the experience of a collaboration with TED-Ed creating climate-related animated video lectures since 2020 in co-production with the Denmark-based Sci-Vi Initiative and The Animation Workshop/VIA UC. To fulfill this aim the research will deliver a State of the Art Report about the current usage of functional animation in research articles and create a systematic review about how the articles describe verbally and visually the used animated materials. The systematic review aims to point out the gaps about how overlooked the animation development process is. The creation of guiding principles can generate discourse between research scientists and animation professionals to establish a common language. This proposal wishes to continue to research on the shift in these articles from 'whether animation facilitates learning' to 'which animation facilitates learning' and bring it further by defining 'how animation collaboration can be integrated into research education'. The spread of animation in education and public science dissemination has exploded due to the technical development of the last 10 years. It has been given a dominant role not only in classroom curricula but also in online education, reinforced by the 2020 COVID-19 pandemic. The theoretical study of the role of animation in learning has undergone incredible change over the past 20 years. The role of animation is shifting from an applied art to a collaborative partner in research dissemination. Practice-oriented research is planned to test and fine-tune the hypothesis through three case studies, that the integration of collaboration between animators and scientists to produce visual research dissemination would not just increase the level of public understanding but help scientists to structure the communication about their research process. The three case studies are designed to establish the integration of animation collaboration into research education. Case Study one is a plan of a crash course for researchers to learn about animated visual communication processes by preparing a plan of an animated explainer. Case Study two is a test ride on how a research institute provides animation collaboration opportunities to allow the researchers to communicate their research findings. Case Study three is a collaboration opportunity between animators and scientists to work with local communities explaining their research about Climate Change and creating visual dissemination using animation. Climate Change, part of the multidisciplinary science of environmental studies, allows research about a complex problem group. All three case studies are aimed to emphasize the creative and productive power of collaboration between visual storytellers and research scientists. The locations and collaboration partners are not yet fixed; exploratory experiments will be performed over the autumn of 2021 to establish the specificities of these data collection contexts. 